\title{
Dynamical polarization potential due to the excitation of collective states
}

\author{
M. V. Andrés, ${ }^{(1)}$ F. Catara, ${ }^{(2,3)}$ and E. G. Lanza ${ }^{(3)}$ \\ ${ }^{(1)}$ Departamento de Física Atómica y Nuclear, Universidad de Sevilla, 41080 Sevilla, Spain \\ ${ }^{(2)}$ Dipartimento di Fisica dell'Università, 95129 Catania, Italy

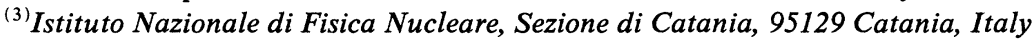

(Received 21 March 1991)

\begin{abstract}
Within the Feshbach formalism we calculate the nucleus-nucleus dynamical polarization potential arising from the coupling of the elastic channel to the collective vibrational states, described by the random-phase approximation. Calculations for the systems ${ }^{16} \mathrm{O}+{ }^{40} \mathrm{Ca}$ and ${ }^{40} \mathrm{Ca}+{ }^{40} \mathrm{Ca}$ show the importance of the high-lying states. They give the main contribution to the real part of the polarization potential at every incident energy, while they dominate the imaginary part only at very high energy. At low energy the absorption is given by the low-lying states. The real and imaginary parts of the potential are shown to obey a dispersion relation. Calculations of elastic cross section give a good description of the experimental data.
\end{abstract}

\section{INTRODUCTION}

The elastic scattering of nuclei is well described by the optical potential. Its real part is usually constructed by means of the double-folding model [1]. The imaginary part describes the depopulation of the elastic channel due to its coupling to the nonelastic ones. This coupling generates also a correction to the real part. Recently, a renewed interest in this so-called dynamical polarized potential has been raised by the experimental evidence [2] of a strong energy dependence of both real and imaginary parts of the optical potential at energies close to the Coulomb barrier, which is known as threshold anomaly. A dispersion relation has been used $[3,4]$ to relate the strong increase of the real part to the decrease of the imaginary one. Thus it is interesting to study the behavior of the polarization potential with the energy within a microscopic approach.

Semiclassical models have been proposed in order to calculate the polarization potential by including lowlying collective vibrational states and one nucleon transfer [5], only one nucleon transfer [6], and both lowand high-lying collective vibrational states [7]. Although the potentials so obtained give a good description of the experimental elastic cross section, a drawback of these models is that the polarization potential is not constructed directly but only by making approximations on quantities expressed as integrals along classical trajectories.

The polarization potential can be calculated in a complete quantum way through the use of the Feshbach formalism [8]. This approach has been exploited by Vinh Mau by using the closure approximation [9]. The main advantage of this method is its simplicity. One can obtain the polarization potential without detailed information on the structure of the nonelastic channels. At the same time, one limitation of the model is its inability to describe how specific channels contribute to the total polarization potential.

In this paper we calculate, following in spirit the ap- proach of Vinh Mau, the polarization potential taking explicitly into account the effects of the excitations of the collective vibrational states, described within the random-phase approximation (RPA). An analysis of the real and imaginary parts of the polarization potential in terms of the single collective modes shows the importance of the giant quadrupole resonance (GQR) states. They give a strong contribution to the imaginary part only at high incident energies, while the real part is dominated by the high-lying states at all energies.

We show that the energy dependence of the real and imaginary parts of the polarization potential follows the trend of the experimental data and satisfies a dispersion relation. Finally, calculations of elastic cross section performed with our microscopic optical potential give a good description of the experimental data. Preliminary results of this model have been presented elsewhere [10].

\section{THE MODEL}

An elegant and transparent way to take into account the coupling of the elastic channel to the nonelastic ones is through the use of the Feshbach formalism [8]. In this approach the effective heavy-ion interaction is written as

$$
\begin{aligned}
V\left(\mathbf{R}, \mathbf{R}^{\prime}\right)= & \langle 00|v(\mathbf{R})| 00\rangle \delta\left(\mathbf{R}-\mathbf{R}^{\prime}\right) \\
& +\sum_{K_{1} K_{2}}\left\langle 00|v(\mathbf{R})| K_{1} K_{2}\right\rangle \\
& \quad \times G_{K_{1} K_{2}}\left(\mathbf{R}, \mathbf{R}^{\prime}\right)\left\langle K_{1} K_{2}\left|v\left(\mathbf{R}^{\prime}\right)\right| 00\right\rangle \\
= & V_{F}(\mathbf{R})+\Delta \mathcal{V}\left(\mathbf{R}, \mathbf{R}^{\prime}\right) .
\end{aligned}
$$

In the first term the nucleon-nucleon interaction $v(\mathbf{R})$ is double folded with the ground-state densities of the two nuclei. In the second term the sum is over all the nonelastic channels, and $v(\mathbf{R})$ is double folded with the transition densities of the two nuclei: it describes the coupling of the elastic channel to the nonelastic ones. This term is the so-called dynamical polarization potential. Its physi- 
cal meaning is transparent from Eq. (1): the interaction acting at the distance $\mathbf{R}^{\prime}$ takes the system into one of the eliminated channels; then it is propagated at another distance $\mathbf{R}$ where the interaction, acting again, brings the system back into the elastic channel. Then the polarization potential is nonlocal, and if one or more of the eliminated channels is open it is also complex and its absorptive part describes the loss of flux from the elastic channel. The coupling also make $\Delta \mathcal{V}$ energy dependent because of the appearance of the energy in the propagator $G_{K_{1} K_{2}}\left(\mathbf{R}, \mathbf{R}^{\prime}\right)$ :

$G_{K_{1} K_{2}}\left(\mathbf{R}, \mathbf{R}^{\prime}\right)=\int d^{3} \mathbf{k} \frac{\varphi_{k}(\mathbf{R}) \varphi_{k}^{*}\left(\mathbf{R}^{\prime}\right)}{E_{\text {c.m. }}-E_{K_{1}}-E_{K_{2}}-\hbar^{2} k^{2} / 2 \mu+i \eta}$,

where $E_{\text {c.m. }}$ is the center-of-mass incident energy, $E_{K_{1}}$ and $E_{K_{2}}$ are the excitation energies of nucleus 1 and 2, respectively, and $\mu$ is the reduced mass. The $\varphi_{k}(\mathbf{R})$ 's are the relative motion wave functions of the colliding nuclei.

The calculation of Eq. (1) is a very difficult task because of the presence of $G_{K_{1} K_{2}}$. We calculate the propagator in the WKB approximation, which has been shown to be a good approximation for $\alpha$-nucleus scattering [11] at energies greater than the Coulomb barrier. In this approximation we have

$$
G_{K_{1} K_{2}}(\rho, s) \simeq-\frac{\mu}{2 \pi \hbar^{2}} \frac{\exp \left[i M_{K_{1} K_{2}}(\rho) s\right]}{s},
$$

with

$M_{K_{1} K_{2}}^{2}(\rho)=\frac{2 \mu}{\hbar^{2}}\left[E_{\text {c.m. }}-E_{K_{1}}-E_{K_{2}}-V_{L}(\rho)-V_{C}(\rho)\right]$,

where

$$
\boldsymbol{\rho}=\frac{1}{2}\left(\mathbf{R}+\mathbf{R}^{\prime}\right), \quad \mathbf{s}=\left|\mathbf{R}-\mathbf{R}^{\prime}\right| .
$$

The local optical potential $V_{L}(\rho)$ is given by

$$
V_{L}(\rho)=V_{F}(\rho)+\Delta \mathcal{V}_{L}(\rho),
$$

while $V_{C}$ is the Coulomb potential between the two nuclei. A procedure to define the local polarization potential $\Delta \mathcal{V}_{L}$ will be given later on in this paper.

In order to make more transparent the contribution of the single terms in Eq. (1), we can separate the summation over $K_{1}, K_{2}$ into three parts,

$$
\sum_{K_{1}, K_{2}}=\sum_{K_{1}=0, K_{2} \neq 0}+\sum_{K_{1} \neq 0, K_{2}=0}+\sum_{K_{1} \neq 0, K_{2} \neq 0}
$$

which give rise to three different contributions to $\Delta \mathcal{V}$,

$$
\Delta \mathcal{V}=\Delta \mathcal{V}_{2}+\Delta \mathcal{V}_{1}+\Delta \mathcal{V}_{12}
$$

respectively. In the first term nucleus 1 stays in its ground state while nucleus 2 is excited, vice versa in the second term, while in the third one both nuclei are excited. This last term is expected to give a small contribution and will be neglected. Then what we have to calculate are terms of the form

$$
\Delta \mathcal{V}_{1}=\sum_{K_{1} \neq 0}\left\langle 00|v(\mathbf{R})| K_{1} 0\right\rangle G_{K_{1} 0}\left(\mathbf{R}, \mathbf{R}^{\prime}\right)\left\langle K_{1} 0\left|v\left(\mathbf{R}^{\prime}\right)\right| 00\right\rangle
$$

The closure approximation model of Vinh Mau consists of replacing the excitation energies $E_{K_{1}}$ and $E_{K_{2}}$ by average values $E_{1}$ and $E_{2}$, respectively. In this case the propagator $G$ does not depend on the particular excitation and it can be taken out of the summation of Eq. (8); then a closure relation over the states $K_{1}$ and $K_{2}$ can be used. This approximation is very good at very high incident energies, while at low energies the values of $M^{2}(\rho)$ [Eq. (3)] can be quite different depending on the values of $E_{K_{1}}$ and $E_{K_{2}}$. Furthermore, the effective nucleon-nucleon interaction is approximated by a separable force.

We want to calculate the polarization potential arising from the excitation of the collective vibrational states and to study the relative importance of low-lying and highlying ones. Thus we do not make use of the above approximations and we explicitly sum over the relevant states, whose energies and transition densities are calculated microscopically within the RPA.

Within the double-folding approach the form factors can be written as

$$
\begin{aligned}
F_{K_{1} 0}(\mathbf{R}) & \equiv\left\langle 00|v(\mathbf{R})| K_{1} 0\right\rangle \\
& =\int d \mathbf{r}_{1} d \mathbf{r}_{2} \rho_{K_{1} 0}\left(\mathbf{r}_{1}\right) v\left(\left|\mathbf{r}_{1}-\mathbf{r}_{2}+\mathbf{R}\right|\right) \rho_{00}\left(\mathbf{r}_{2}\right) .
\end{aligned}
$$

The explicit expression of the radial part of the transition density $\rho_{0 K_{1}}\left(\mathbf{r}_{1}\right)$ is

$$
\begin{aligned}
\rho_{K_{1} 0}(r)=\frac{1}{\sqrt{4 \pi}} \sum_{p h} & (-)^{j_{h}+1 / 2}\left(j_{p}-\frac{1}{2} j_{h} \frac{1}{2} \mid L_{1} 0\right) \\
& \times \frac{\hat{j}_{p} \hat{j}_{h}}{\hat{L}_{1}}\left(X_{p h}^{K_{1}}-Y_{p h}^{K_{1}}\right) R_{p}(r) R_{h}(r)
\end{aligned}
$$

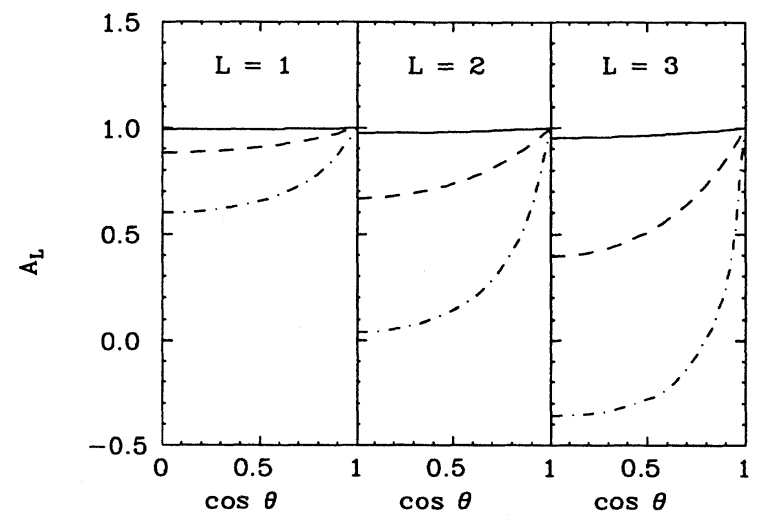

FIG. 1. The quantity $A_{L}$, defined in Eq. (11), as function of $\cos \theta$ for three values of the angular momentum $L$. In each sector the three curves correspond to a fixed value of $\rho=8 \mathrm{fm}$ and to the following values of $s: 1 \mathrm{fm}$ (solid line), $4 \mathrm{fm}$ (dashed line), and $8 \mathrm{fm}$ (dot-dashed line). The results corresponding to odd values of $L$ are multiplied by -1 . 
where $R_{p}\left(R_{h}\right)$ is the particle (hole) wave function; $X_{p h}^{K_{1}}$ and $Y_{p h}^{K_{1}}$ are the forward and backward RPA amplitudes corresponding to the mode $K_{1}$ whose multipolarity is $L_{1}$.

The calculation of $\Delta \mathcal{V}_{12}(\rho, s)$ is reported in the Appendix. Expressions for it and for $\Delta \mathcal{V}(\rho, s)$ are given by Eqs.
(A8) and (A9), respectively. Their angular part can be easily worked out if we assume that the potential $\Delta \mathcal{V}$ is weakly dependent on the angle $\theta$ between $\rho$ and $\mathbf{s}$. This has been shown to be true for nucleon-nucleus scattering [12]. The angular part of $\Delta \mathcal{V}_{1}$ can be written as

$$
\begin{aligned}
A_{L}(\rho, s, x)= & \left(\sqrt{\rho^{2}+\frac{1}{4} s^{2}+\rho s x} \sqrt{\rho^{2}+\frac{1}{4} s^{2}-\rho s x}\right)^{-L} \rho^{2 L} \\
& \times \sum_{\lambda, \lambda^{\prime}=0}^{L}(-)^{\lambda^{\prime}}\left[\frac{s}{2 \rho}\right]^{\lambda+\lambda^{\prime}}[2(L-\lambda)+1]^{1 / 2}\left[2\left(L-\lambda^{\prime}\right)+1\right]^{1 / 2}\left[\begin{array}{c}
2 L+1]^{1 / 2}\left[\begin{array}{c}
2 L+1 \\
2 \lambda
\end{array}\right]^{1 / 2} \\
2 \lambda^{\prime}
\end{array}\right. \\
& \times \sum_{f=\left|\lambda-\lambda^{\prime}\right|}^{\lambda+\lambda^{\prime}}(-)^{f} W\left(\lambda, L-\lambda, \lambda^{\prime}, L-\lambda^{\prime} ; L f\right)\left\langle L-\lambda 0 L-\lambda^{\prime} 0 \mid f 0\right\rangle\left\langle\lambda 0 \lambda^{\prime} 0 \mid f 0\right\rangle P_{f}(x) .
\end{aligned}
$$

where $x=\cos \theta$ and $\theta$ is the angle between $\rho$ and $s$. Numerical calculations of Eq. (11) show that the variation of $A_{L}$ with $\theta$ may be very different depending on the values of $\rho$ and $s$. In particular, we show in Fig. 1 the behavior of $A_{L}$, for $\rho=8 \mathrm{fm}$ and $s=1,4,8 \mathrm{fm}$, as function of $\cos \theta$ for three values for $L$. We see that a rapid variation with $\cos \theta$ is found for $\rho=s=8 \mathrm{fm}$, while for small values of $s$ the quantity $A_{L}$ is almost independent of $\cos \theta$. This range of values of large $\rho$ and small $s$ is the physically interesting domain, where the localization procedure that we will describe later on also makes sense. So in this range of values $\Delta \mathcal{V}$ is weakly dependent on the angle $\theta$. Choosing the value $\cos \theta=1$, which corresponds to take the maximum contribution, we have

$$
\begin{aligned}
\left.A_{L}(\rho, s, x)\right|_{x=1}=\left(\rho^{2}-\frac{1}{4} s^{2}\right)^{-L} \sum_{\lambda=0}^{L} & {\left[\frac{s}{2}\right]^{\lambda} \rho^{L-\lambda}\left(\begin{array}{c}
2 L+1 \\
2 \lambda
\end{array}\right]^{1 / 2}\langle\lambda 0 L 0 \mid L-\lambda \quad 0\rangle(-)^{\lambda} } \\
& \times \sum_{\lambda^{\prime}=0}^{L}\left[\frac{s}{2}\right]^{\lambda^{\prime}} \rho^{L-\lambda^{\prime}}\left(\begin{array}{c}
2 L+1 \\
2 \lambda^{\prime}
\end{array}\right)^{1 / 2}\left\langle\lambda^{\prime} 0 L \quad 0 \mid L-\lambda^{\prime} 0\right\rangle=(-)^{L} .
\end{aligned}
$$

Then the potentials $\Delta \mathcal{V}_{12}$ and $\Delta \mathcal{V}_{1}$ can be written as

$$
\Delta \mathcal{V}_{12}(\rho, s)=\frac{1}{(2 \pi)^{6}} \sum_{K_{1}, K_{2}} G_{K_{1} K_{2}}(\rho, s) \hat{L}_{1}^{2} \hat{L}_{2}^{2} \sum_{J} \mathcal{R}_{K_{1} K_{2}}^{J}(\rho, s) \mathcal{R}_{K_{1} K_{2}}^{J}(\rho,-s)\left\langle L_{1} \quad 0 L_{2} 0 \mid J \quad 0\right\rangle^{2}
$$

and

$$
\Delta \mathcal{V}_{1}(\rho, s)=\frac{1}{16 \pi^{5}} \sum_{K_{1}} G_{K_{1} 0}(\rho, s) \mathcal{R}_{K_{1} 0}^{L_{1}}(\rho, s) \mathcal{R}_{K_{1} 0}^{L_{1}}(\rho,-s) \hat{L}_{1}^{2}
$$

where $\mathcal{R}_{K_{1} K_{2}}^{J}(\rho, s)$ is defined in Eq. (A7) of the Appendix.

The potential calculated with just the described procedure is nonlocal. But, since it is always preferable to use local potentials, at least for the calculation of physical observables, we use a standard procedure [13] to obtain a local potential from a nonlocal one. This procedure is applicable whenever the range of nonlocality of $\Delta \mathcal{V}$ is small with respect to its radius. Thus, we have

$$
\begin{aligned}
& \Delta V(\rho)=4 \pi \int j_{0}(k s) \operatorname{Re} \Delta \mathcal{V}(\rho, s) s^{2} d s \\
& W(\rho)=4 \pi \int j_{0}(k s) \operatorname{Im} \Delta \mathcal{V}(\rho, s) s^{2} d s
\end{aligned}
$$

where

$$
k=\frac{2 \mu}{\hbar^{2}}\left[E_{\mathrm{c} . \mathrm{m} .}-V_{F}(\rho)-V_{C}(\rho)\right]
$$

It has been shown [9] that the hypothesis over the range of nonlocality of $\Delta \mathcal{V}$ is valid, at least for large values of $\rho$. Indeed, in Fig. 2 we show the real and imaginary parts of Eq. (14) as functions of $s$ for two fixed values of $\rho$. For large values of $\rho$ it is found that the range of nonlocality is about $1 \mathrm{fm}$. This value is consistent with previous ones given in the literature $[9,14]$. Thus the hypothesis of independence of $\Delta \mathcal{V}$ on the angle between $\rho$ and $\mathbf{s}$, discussed before, is also justified.

\section{RESULTS AND DISCUSSION}

We have done calculations for the systems ${ }^{16} \mathrm{O}+{ }^{40} \mathrm{Ca}$ and ${ }^{40} \mathrm{Ca}+{ }^{40} \mathrm{Ca}$ at several incident energies. The levels used in the calculations are reported in Table I. They have been obtained with a self-consistent RPA code using an SGII force [15]. We have included all the states which exhaust at least $10 \%$ of the energy-weighted sum rule 

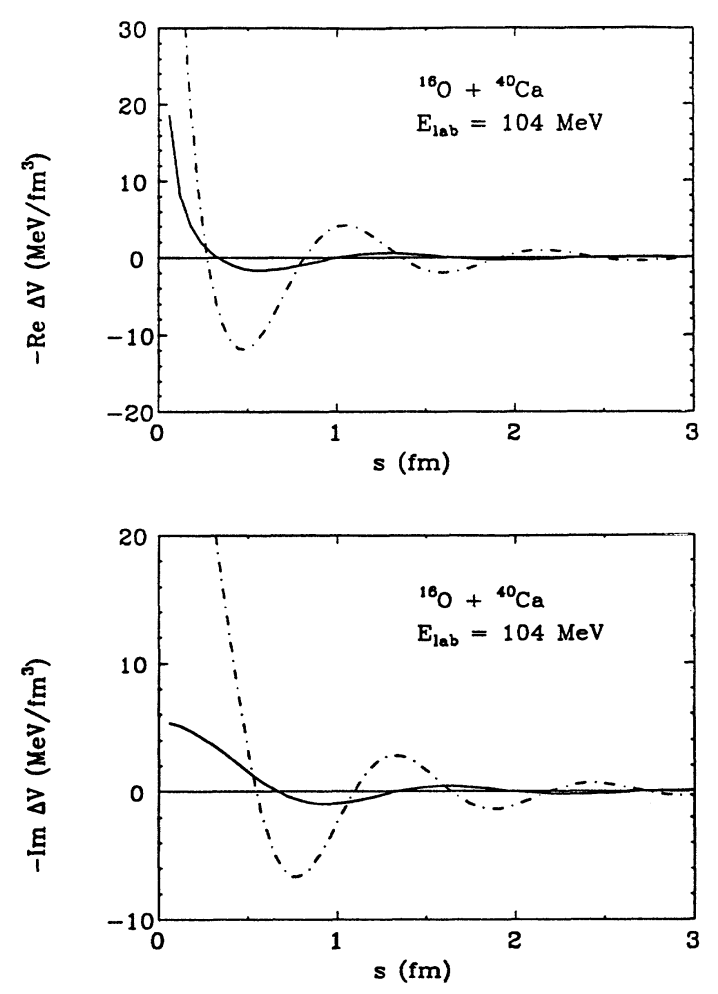

FIG. 2. Real and imaginary parts of the nonlocal polarization potential $\Delta \mathcal{V}_{1}(\rho, s)$, for the system ${ }^{16} \mathrm{O}+{ }^{40} \mathrm{Ca}$ at $E_{\text {lab }}=104$ $\mathrm{MeV}$, as a function of $s$ for two fixed values of $\rho: 7 \mathrm{fm}$ (dashed line) and $8 \mathrm{fm}$ (solid line).

(EWSR). The RPA calculations have been done with a large number of particle-hole configurations $(\sim 350$ for the $3^{-}$of ${ }^{40} \mathrm{Ca}$ ) in order to get a good description of the low-lying states [16]. Both energies and transition densities compare well with the experimental data. The effective M3Y nucleon-nucleon interaction has been used

TABLE I. Properties of the RPA states used in the calculations.

\begin{tabular}{lllrr}
\hline \hline Nuclei & $J^{\pi}$ & $T$ & $E^{*}(\mathrm{MeV})$ & \%EWSR \\
\hline${ }^{16} \mathrm{O}$ & $1^{-}$ & 1 & 19.096 & 27 \\
& $1^{-}$ & 1 & 19.996 & 21.4 \\
& $2^{+}$ & 0 & 19.945 & 44 \\
& $2^{+}$ & 0 & 20.620 & 13 \\
& $2^{+}$ & 0 & 21.280 & 12.6 \\
& $3^{-}$ & 0 & 7.220 & 8.8 \\
& $3^{-}$ & 0 & 32.870 & 8.4 \\
& $3^{-}$ & 0 & 34.610 & 8.9 \\
& & & & \\
${ }^{40} \mathrm{Ca}$ & $1^{-}$ & 1 & 16.302 & 9.8 \\
& $1^{-}$ & 1 & 16.765 & 15.2 \\
& $1^{-}$ & 1 & 17.372 & 10.6 \\
& $1^{-}$ & 1 & 17.883 & 10.6 \\
& $1^{-}$ & 1 & 18.296 & 16 \\
& $1^{-}$ & 1 & 18.632 & 80 \\
& $2^{+}$ & 0 & 16.741 & 15 \\
& $3^{-}$ & 0 & 4.830 & \\
& $3^{-}$ & 0 & 31.281 & \\
& & & & \\
\hline \hline
\end{tabular}

in order to construct the double-folding potential $V_{F}$ of Eq. (1) and the form factors of Eq. (9).

In order to study the interplay between the low-lying and high-lying states we have made an analysis in terms of the single vibrational collective states. In the left part of Fig. 3 we show the real and imaginary parts of the polarization potential for the system ${ }^{40} \mathrm{Ca}+{ }^{40} \mathrm{Ca}$ at $E_{\text {lab }}=240 \mathrm{MeV}$. Each line corresponds to the contribution due to different multipolarities as indicated in the figure; the total potential is given by the solid line. The relative contribution of the single collective states can be better seen in the right part of Fig. 3, where for the same system we show the real and imaginary parts of the polarization plotted in percentage of the total potential as function of the relative distance $R$. Each line corresponds to the relative contribution of the different multipolarities, as indicated in the figure. We note that the absorptive part is given almost completely by the lowlying $3^{-}$state. Conversely, the contribution of low-lying and GQR states to the real part $\Delta V$ is of the same magnitude. The high-lying $3^{-}$states and the giant dipole resonance states give essentially zero contribution. For the dipole isovector state this contribution comes only through the small mixture of $T=0$ components due to the Coulomb interaction. This result is not peculiar of the particular system: in general, at low energy the absorptive part of the optical potential is given by the lowlying states, while both low and GQR states contribute to the real part. In fact, also for the system ${ }^{16} \mathrm{O}+{ }^{40} \mathrm{Ca}$ at $E_{\text {lab }}=104 \mathrm{MeV}$ (see upper part of Fig. 4) a similar behavior is found.

In order to investigate the polarization potential at energies much higher than the Coulomb barrier we have calculated $\Delta V$ and $W$ for the system ${ }^{16} \mathrm{O}+{ }^{40} \mathrm{Ca}$ at several incident energies. In Fig. 4 we show the relative contribution of the single modes to the real (left) and imaginary (right) part of the polarization potential as a function of $R$ and for three different values of the incident energy. Again, the multipolarities are indicated for each curve. We note two striking features. (1) The real part is dominated by the GQR states which give, at high energy, $80 \%$ of the potential. The contribution of the low-lying $3^{-}$state, comparable in the peripheral region to the $2^{+}$ states, is decreasing when the energy increases. The high-lying $3^{-}$states and the GDR states behave in an opposite fashion. However, they never reach important values. (2) Conversely, the behavior of the absorptive part changes drastically, going from 104 to $640 \mathrm{MeV}$. In fact, as we have seen before, at low energy the imaginary potential is essentially due to the $3^{-}$low-lying states, while as the energy increases the dominant absorption comes from the GQR of both nuclei. This behavior can be understood in semiclassical terms [7]; higher energies correspond to shorter interaction times; hence, by the uncertainty relations, the probability of excitation of highlying states is higher. This mechanism does not work in the case of the real part because the process involved in the formation of $\Delta V$ is a virtual one.

A deeper analysis shows that the major part of the total $3^{-}$contribution comes from the low-lying state of the ${ }^{40} \mathrm{Ca}$. This can be seen in Fig. 5, where for the system 
${ }^{16} \mathrm{O}+{ }^{40} \mathrm{Ca}$ at $E_{\mathrm{lab}}=104 \mathrm{MeV}$ we show how the polarization potential due only to the low-lying states is distributed between the two partners of the reaction. We see that the heavier partner is responsible for most of the effect. This trend does not change at higher energies; the only difference is that, for instance at $E_{\text {lab }}=640 \mathrm{MeV}$, the two curves get closer for large $R$. On the contrary, for the high-lying states it is the lighter partner which gives the greater contribution, although the difference is not as big as in the previous case. As an example, we show in Fig. 6 the polarization potential due to the GQR states for the case of ${ }^{16} \mathrm{O}+{ }^{40} \mathrm{Ca}$ at $E_{\text {lab }}=640 \mathrm{MeV}$.

In order to check the range of validity of the previous results with respect to the relative importance of GQR and low-lying states, we have done calculations for laboratory energies from 10 to $320 \mathrm{MeV}$ for the system ${ }^{16} \mathrm{O}+{ }^{40} \mathrm{Ca}$ at a fixed distance $R=9 \mathrm{fm}$. The results are reported in Fig. 7, where the real and imaginary parts of the polarization potential are plotted as functions of the energy. Their energy dependence resembles very much the experimental findings of Ref. [2]. The dashed line is a result of a calculation done including only the low-lying $3^{-}$states of the two nuclei; the solid line has been obtained by using all the states of Table I. As was expected, at low energy the absorptive part is given only by the $3^{-}$ states, the contribution of the high-lying states increasing with the energy until reaching a maximum around 650 $\mathrm{MeV}$ (not shown in the figure) and then decreasing very slowly to 0 .
Surprisingly enough, for the real part we have a completely different interplay between low- and high-lying states: the latter are giving a big contribution even at very low energy. The different behavior depends on the physical process giving rise to the real and imaginary parts of the polarization potential. The latter is due to real excitation of the nuclei, and thus it vanishes at low incident energy when the channels are closed. The real part is due to virtual excitation of the nuclei, so it is present at all energies. Previous calculations of the polarization potential have not taken into account the GQR states, missing in this case at least $50 \%$ of the effect.

The energy dependence of the polarization potential, shown in Fig. 7, has a twofold origin. The dynamic energy dependence is due to the appearance of the center-ofmass energy in the definition of the propagator. The second one can be thought of as a spurious one [17] because it is due to the localization procedure described in Eqs. (15) and (16) and it comes through the local momentum $k$.

The energy dependence of the real and imaginary parts of the polarization potential are governed by the dispersion relation. This relation is deduced from the general principle of causality, so every polarization potential should obey it. The possible violation of the dispersion relation due to the extra energy dependence introduced by the localization procedure has been found to be small $[9,18,19]$.

In order to check it in our case we use the linear
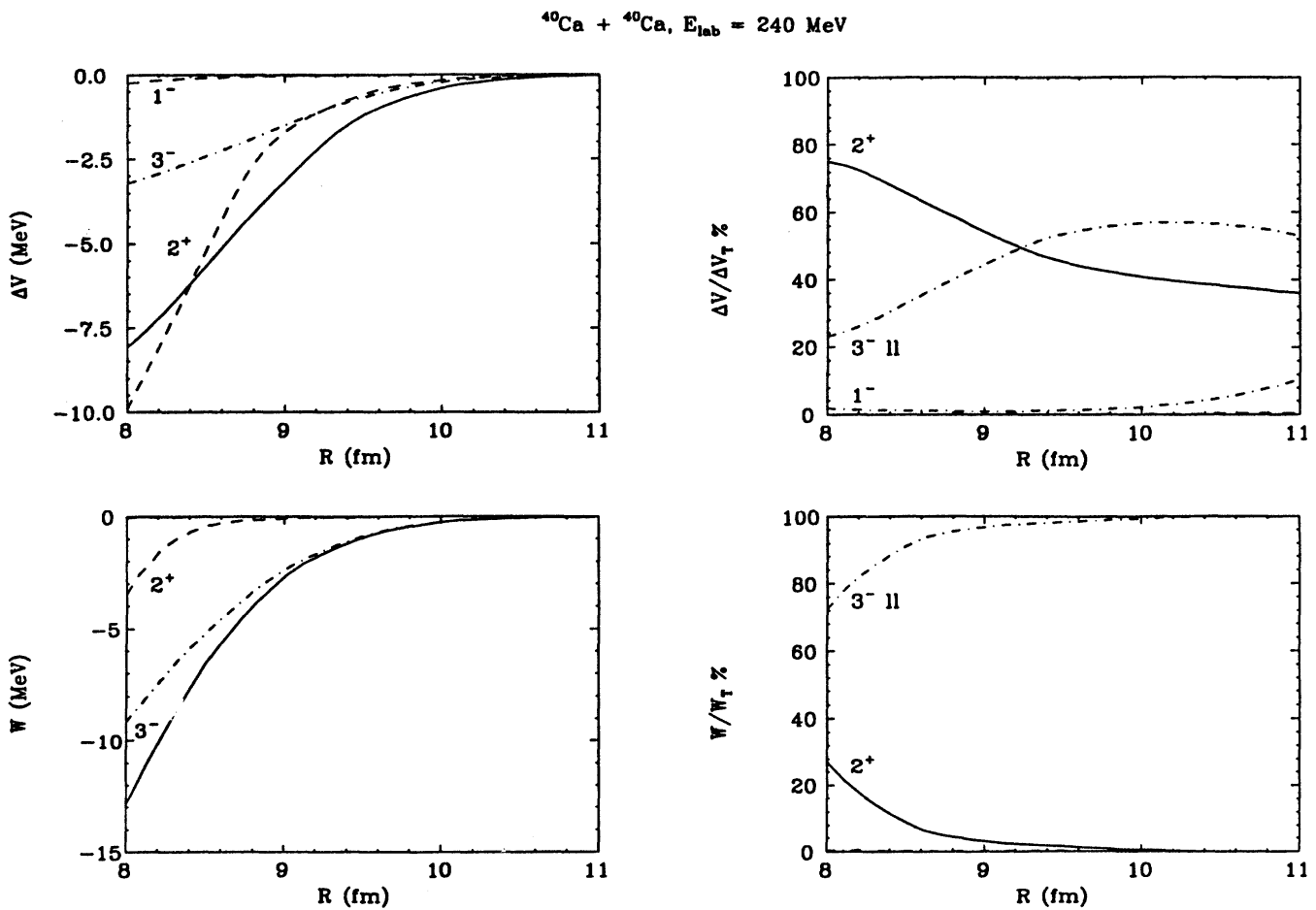

FIG. 3. Real and imaginary parts of the local polarization potential for ${ }^{40} \mathrm{Ca}+{ }^{40} \mathrm{Ca}\left(E_{\mathrm{lab}}=240 \mathrm{MeV}\right)$ as a function of the relative distance $R$. Each line corresponds to the contribution of different multipolarities as indicated in the figure. On the left part the total potential is given by the solid line. On the right part the potentials are plotted in percentage of the total one. 
schematic model given by Mahaux et al. [4]. In particular, we use the model of Eq. (3.15) of Ref. [4], where we have changed the misprinted plus sign of the last term to a minus. In this model the imaginary part is segmented into three parts, and this makes it possible to find an algebraic expression for the corresponding real part through the subtracted dispersion relation. By using Eq. (3.15) of Ref. [4] we have then calculated the real part of the polarization potential for both of the imaginary parts of Fig. 7; namely, the one due to only the $3^{-}$low-lying states and the one calculated with all the states of Table I. In both cases the schematic potential has been normalized to the calculated one at $E_{\text {lab }}=320 \mathrm{MeV}$. The squares in Fig. 8 are the result of this calculation. The agreement with the curves calculated by means of Eq. (15) is very good. This implies that the localization pro- cedure used here is a good one in the sense that it does not violate the dispersion relation, at least at the distance considered in the calculations.

Optical model analysis $[1,20,21]$ on elastic scattering data is available for the system ${ }^{16} \mathrm{O}+{ }^{40} \mathrm{Ca}$. Our imaginary potential shows a behavior in qualitative agreement with the empirical one, namely, it decreases in the $E_{\text {lab }}<80 \mathrm{MeV}$ domain and goes to 0 at $E_{\text {lab }} \sim 40 \mathrm{MeV}$. In the same energy region we have a corresponding increase for $\Delta V$. Unfortunately, the empirical values show a considerable scatter which makes a quantitative comparison hard. As pointed out by the authors of Ref. [4], a consistent reanalysis of all the data would be interesting.

In order to have a further test of our microscopic optical potential we have calculated the elastic cross section for the systems ${ }^{16} \mathrm{O}+{ }^{40} \mathrm{Ca}$ and ${ }^{40} \mathrm{Ca}+{ }^{40} \mathrm{Ca}$ at various en-
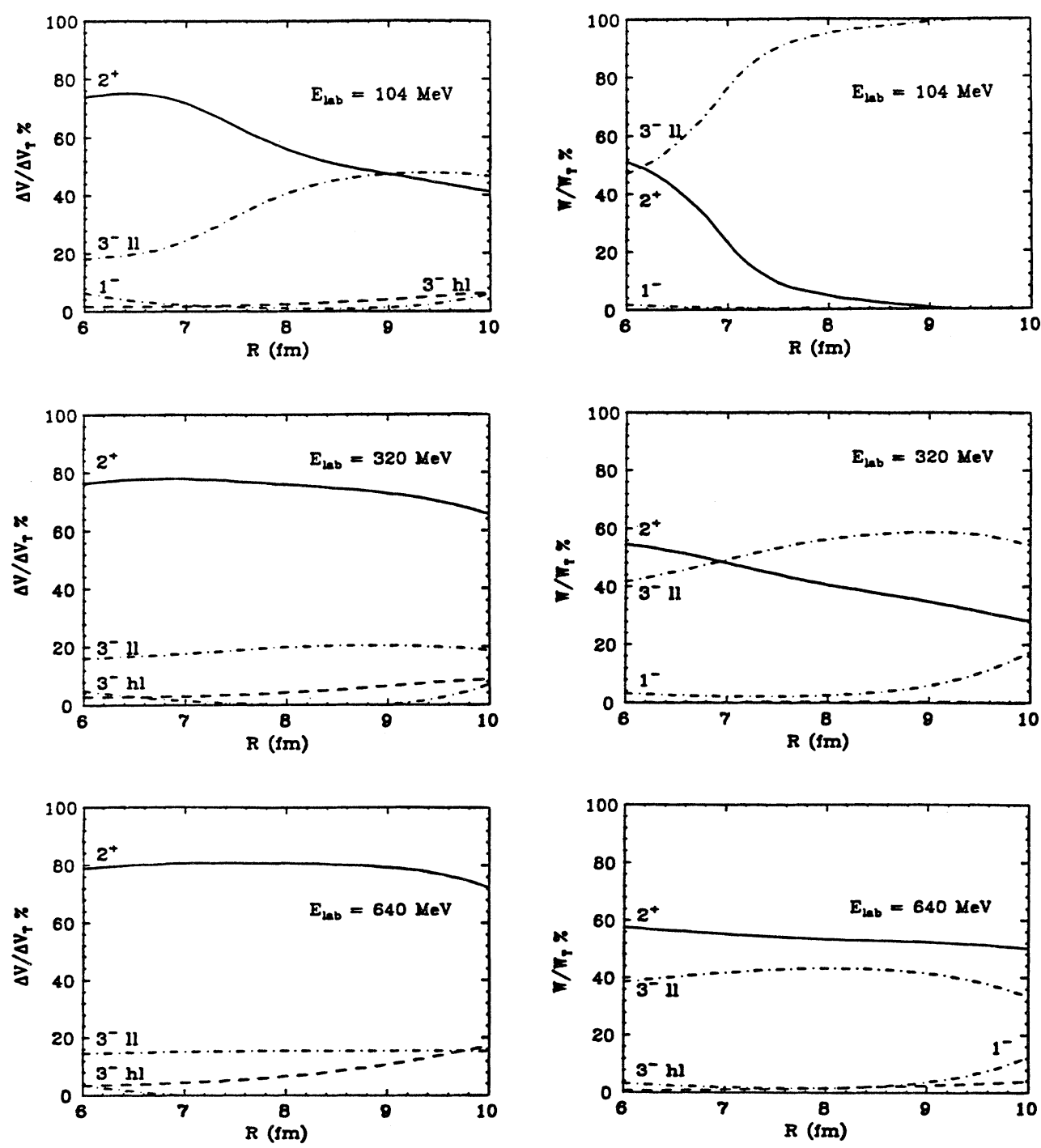

FIG. 4. Real (left) and imaginary (right) parts of the polarization potential for ${ }^{16} \mathrm{O}+{ }^{40} \mathrm{Ca}$ at different values of incident energy, plotted in percentage of the total potential and as a function of the relative distance $R$. 

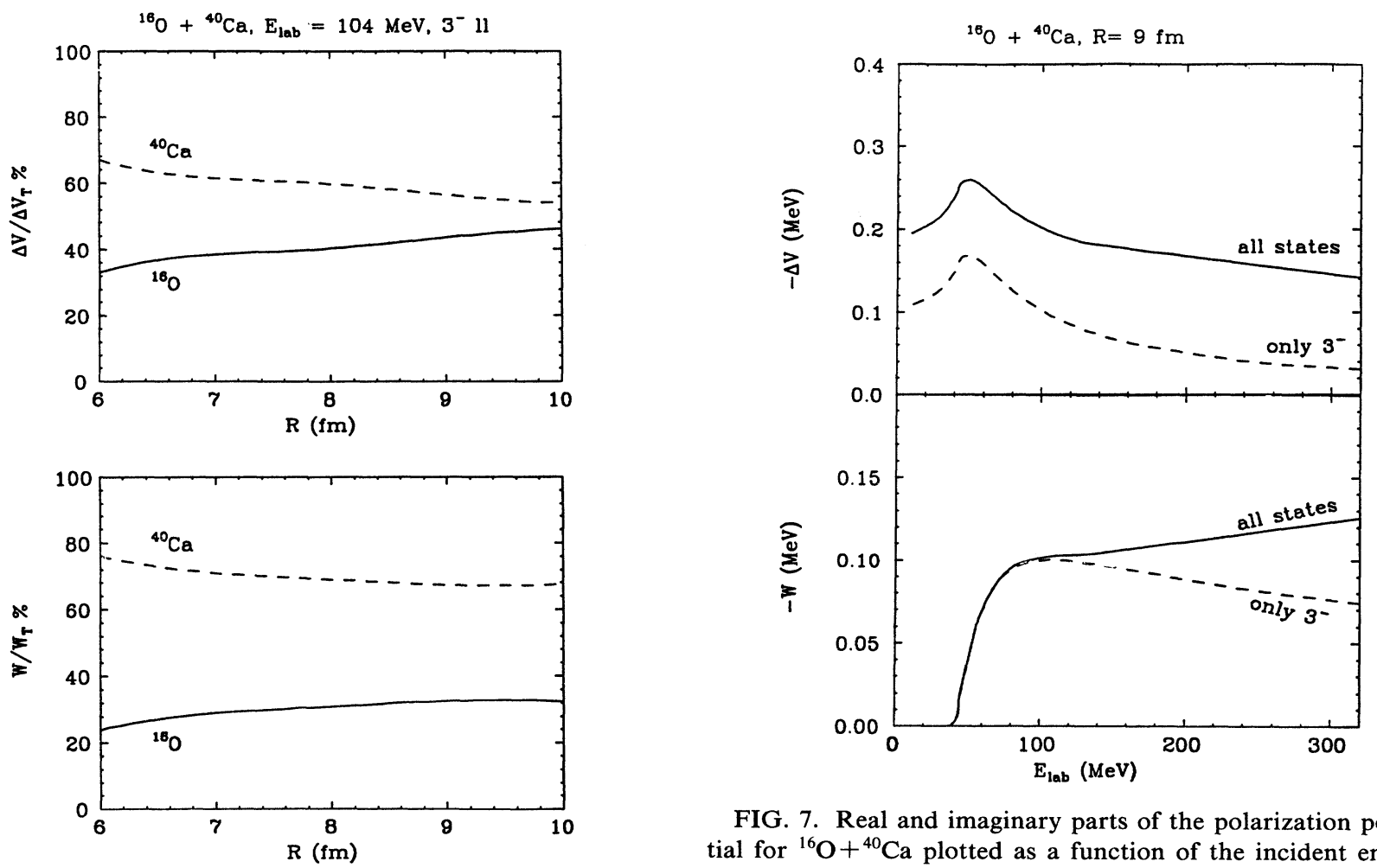

FIG. 5. Real and imaginary parts of the polarization potential for ${ }^{16} \mathrm{O}+{ }^{40} \mathrm{Ca}$ at $E_{\text {lab }}=104 \mathrm{MeV}$, plotted in percentage of the potential given only by the low-lying $3^{-}$states of the two nuclei.
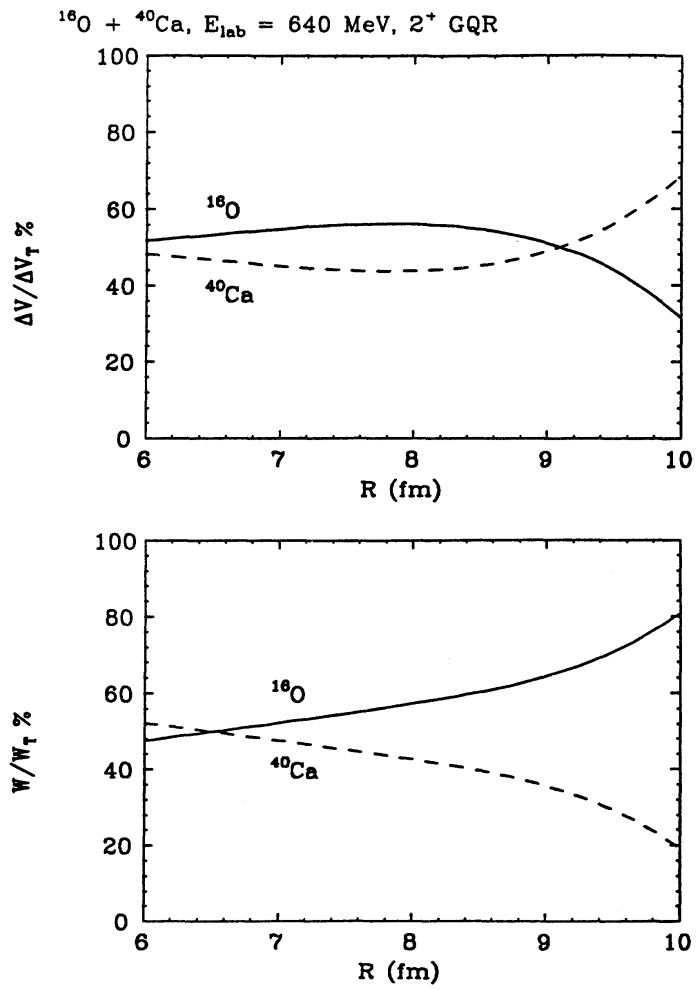

FIG. 6. Same as Fig. 5 at $E_{\text {lab }}=640 \mathrm{MeV}$. Here the polarization potential is due only to the GQR states.

FIG. 7. Real and imaginary parts of the polarization potential for ${ }^{16} \mathrm{O}+{ }^{40} \mathrm{Ca}$ plotted as a function of the incident energy for a fixed value of $R=9 \mathrm{fm}$. The dashed curves refer to a calculation performed by including only the low-lying $3^{-}$states of the two nuclei. The solid lines are the results of a calculation done with all the states of Table I.

ergies. In Fig. 9 are reported the elastic differential cross sections for the cases ${ }^{16} \mathrm{O}+{ }^{40} \mathrm{Ca}$ at $E_{\text {lab }}=104 \mathrm{MeV}$ (upper part) and ${ }^{40} \mathrm{Ca}+{ }^{40} \mathrm{Ca}$ at $E_{\text {lab }}=143 \mathrm{MeV}$ (lower part). The agreement with the data is very good considering the fact that we do not have any adjustable parameter. We are aware of the fact that the nonelastic channels taken into account here to construct the polarization potential are not at all exhaustive. In particular, we have not con-

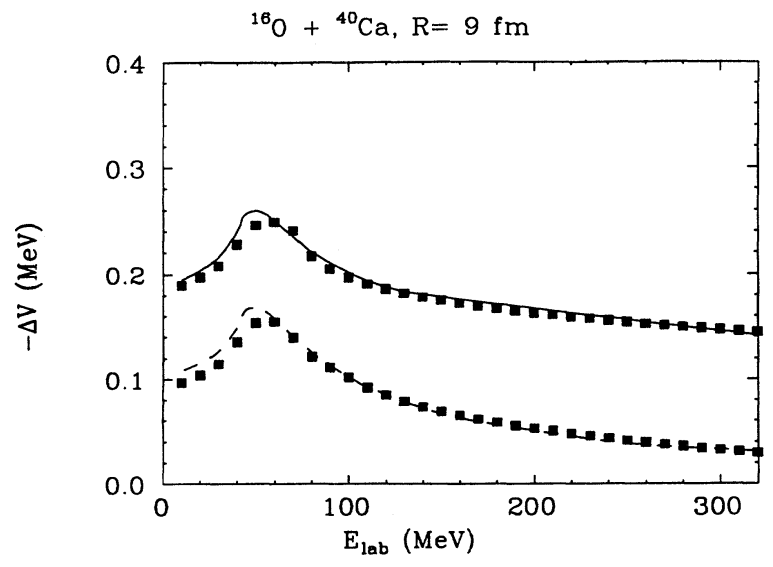

FIG. 8. Same as the lower part of Fig. 7. The squares correspond to a subtracted dispersion relation calculation (see text). 

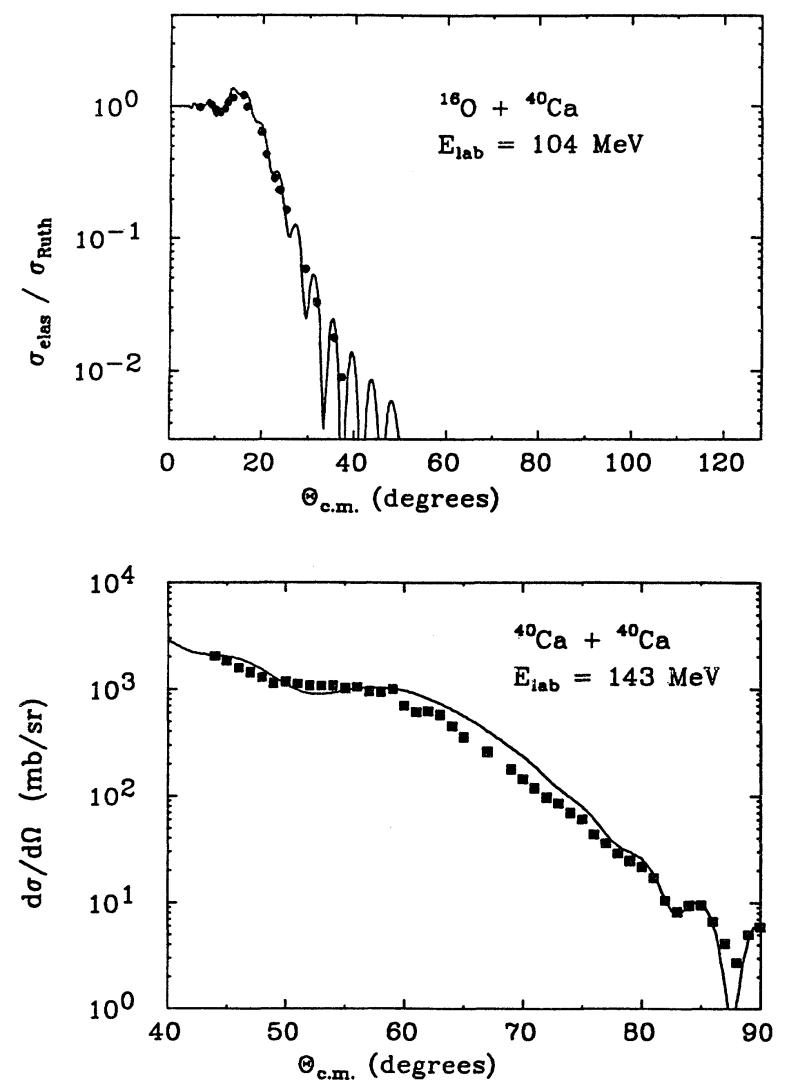

FIG. 9. Elastic scattering angular distribution for ${ }^{16} \mathrm{O}+{ }^{40} \mathrm{Ca}$ and ${ }^{40} \mathrm{Ca}+{ }^{40} \mathrm{Ca}$. The latter is plotted in absolute units. The data are from Refs. [21] and [22], respectively.

sidered the transfer channels which are thought to be important at least for asymmetric systems. This may be the reasons why we have strong oscillations in the elastic cross section at backward angles for the ${ }^{16} \mathrm{O}+{ }^{40} \mathrm{Ca}$ case. In fact, as is shown in Ref. [5], the imaginary potential due to the transfer channels having a longer range may smear out the oscillations.

Another point to be investigated is how much the results depend on the values of the excitation energies of the collective states used in the calculations. In other words, is the use of an average excitation energy in the propagator and then the use of the closure approximation justified, or is it important to use the proper energies for low- and high-lying states? To answer this question we have done a calculation including all the states of Table I, where the energies have been arbitrarily put equal to a fixed value. In particular, we have chosen $\bar{E}_{16}=6.5$ $\mathrm{MeV}$ and $\bar{E}_{40 \mathrm{Ca}}=5.0 \mathrm{MeV}$ (Ref. [9]). The results are reported in Fig. 10 as dashed lines, while the solid lines result from the RPA energies reported in Table I. The behavior with the energy of the imaginary potential is quite different in the two cases. In the calculation corresponding to the solid line the effect of the high-lying states is evident: the potential goes up as the incident energy increases. The dashed line resembles the one in Fig. 7,

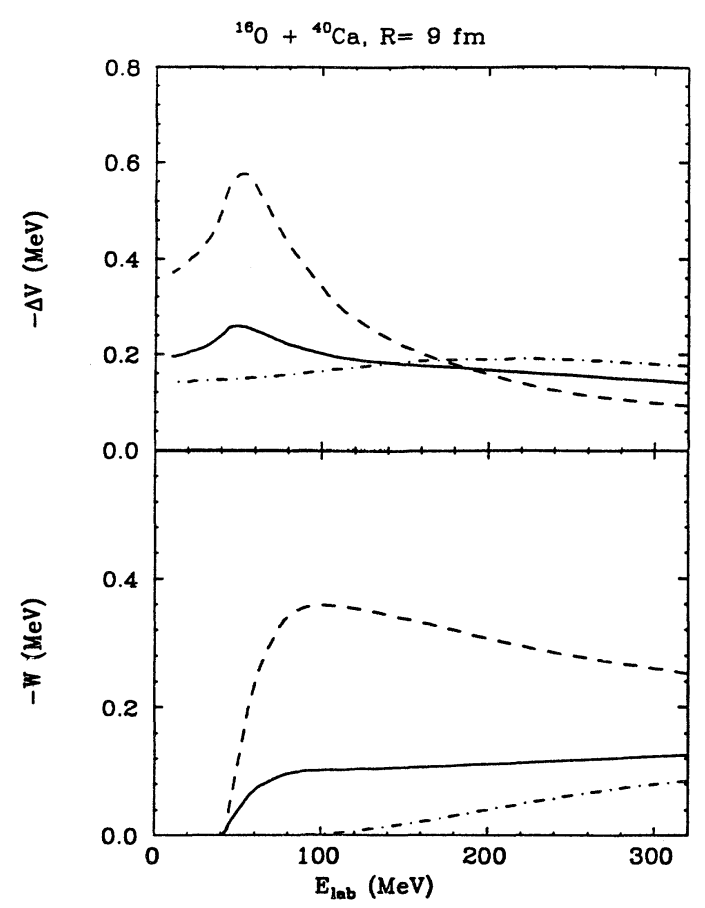

FIG. 10. Same as Fig. 7. The dashed curves refer to a calculation performed with constant excitation energy $\bar{E}_{16_{0}}=6.5$ $\mathrm{MeV}$ and $\bar{E}_{40} \mathrm{Ca}=5.0 \mathrm{MeV}$, while the dot-dashed ones correspond to the values $\bar{E}_{16_{\mathrm{O}}}=20 \mathrm{MeV}$ and $\bar{E}_{40 \mathrm{Ca}}=17 \mathrm{MeV}$ (see text). The solid lines are the same as the ones plotted in Fig. 7.

which was calculated with only the two low-lying states. Thus all the states now give an equally important contribution, at least at low energies. The magnification factor between the two curves is not equal to the number of states used in the calculation because we have kept the RPA form factors in both cases. For completeness we have done a calculation with the average energies close to the energies of the giant quadrupole resonance states, i.e., $\bar{E}_{16 \mathrm{O}}=20 \mathrm{MeV}$ and $\bar{E}_{40 \mathrm{Ca}}=17 \mathrm{MeV}$. The results (dotdashed line in Fig. 10) show an enlargement of the energy scale: the rapid increase and then decrease of the real part of the polarization potential is now spread out over a much larger range of incident energy. As expected, the imaginary part is important at high energies and vanishes at $E_{\text {lab }} \sim 100 \mathrm{MeV}$ (see also lower part of Fig. 7). The real parts are governed by the dispersion relation: different behaviors of the absorption will produce different behaviors of the real polarization potential. In particular, according to the simple form of the linear schematic model of Ref. [4] [Eq. (3.17)], the positions of the maxima of the real parts are determined by the energy value corresponding to half the interval where the imaginary potential goes rapidly to 0 . The fact that the real potential corresponding to the dot-dashed line has a maximum near 200 $\mathrm{MeV}$ confirms this simple model.

We have shown, at least for the two extreme cases presented in Fig. 10, that the use of average excitation energies in the propagator of Eq. (2) gives rise to polariza- 
tion potentials which differ in shape and magnitude from the potential calculated with the proper energies for both low- and high-lying states. Since these results are not at all exhaustive, this problem should be further investigated.

\section{CONCLUSIONS}

Within the Feshbach formalism we have calculated the dynamical polarization potential arising from the coupling of the elastic channel to the collective vibrational states. The latter were constructed within self-consistent RPA with the SGII force. Both energies and transition densities compare well with the available experimental data. The propagator was calculated in the WKB approximation, which is very good at incident energies greater than the Coulomb barrier. The bare nucleusnucleus potential has been constructed by double folding the effective M3Y interaction with the HF densities of the two nuclei. We have not made use of the closure approximation but we have summed over a finite number of relevant states, each one with its own energy. The localization procedure we have used does not destroy the energy dependence of the polarization potential: The real and imaginary parts satisfy the dispersion relation.

We have done an analysis of the real and imaginary parts of the polarization potential in terms of the relative contributions of the single collective states for the systems ${ }^{16} \mathrm{O}+{ }^{40} \mathrm{Ca}$ and ${ }^{40} \mathrm{Ca}+{ }^{40} \mathrm{Ca}$ at several incident energies. As one should expect, at low incident energies the main contribution to the imaginary part of the polarization potential comes from the $3^{-}$low-lying states. As the energy is increased the role played by the $3^{-}$states is taken over by the GQR states which give the main effect. Conversely, the real part is dominated by the high-lying states in all the energy range investigated, and they contribute up to $80 \%$ to $\Delta V$. This novel result shows that the GR states cannot be disregarded in the construction of a dynamical polarization potential.

We have also shown that the use of the average excitation energies, in the two extreme cases treated here, gives rise to a polarization potential which differs appreciably both in shape and magnitude from the one calculated with the proper energies.

The energy dependence of the polarization potential has been checked by means of the dispersion relation. In particular, we have used the linear schematic model of Ref. [4]. The result is very good. Calculations of elastic cross section give a good description of experimental data: these calculations have been done with no adjustable parameters.

\section{ACKNOWLEDGMENTS}

We wish to thank M. A. Nagarajan, N. Van Giai, and N. Vinh Mau for useful discussion and suggestions. We also thank N. Van Giai for making his RPA code available to us.

\section{APPENDIX}

In this Appendix we want to calculate the quantity $\Delta \mathcal{V}_{12}$ which is defined as

$$
\Delta \mathcal{V}_{12}\left(\mathbf{R}, \mathbf{R}^{\prime}\right)=\sum_{K_{1} K_{2}} F_{K_{1} K_{2}}(\mathbf{R}) G_{K_{1} K_{2}}\left(\mathbf{R}, \mathbf{R}^{\prime}\right) F_{K_{1} K_{2}}^{*}\left(\mathbf{R}^{\prime}\right)
$$

where $F_{K_{1} K_{2}}(\mathbf{R})$ is given by

$$
\begin{aligned}
F_{K_{1} K_{2}}(\mathbf{R}) \equiv\left\langle 00|v(\mathbf{R})| K_{1} K_{2}\right\rangle= & \int d \mathbf{r}_{1} d \mathbf{r}_{2} \rho_{K_{1} 0}\left(\mathbf{r}_{1}\right) v\left(\left|\mathbf{r}_{1}-\mathbf{r}_{2}+\mathbf{R}\right|\right) \rho_{0 K_{2}}\left(\mathbf{r}_{2}\right) \\
= & \frac{1}{4 \pi^{5 / 2} \int} d p p^{2} \widetilde{v}(p) \bar{\rho}_{K_{1} 0}(p) \bar{\rho}_{0 K_{2}}(p) i^{L_{1}-L_{2}} \hat{L}_{1} \hat{L}_{2}(-)^{M_{1}+M_{2}} \\
& \quad \times \sum_{j} \frac{i^{-J}}{J} j_{J}(p R)\left\langle L_{1} M_{1} L_{2} M_{2} \mid J M_{1}+M_{2}\right\rangle\left\langle L_{1} 0 L_{2} 0 \mid J 0\right\rangle Y_{J M_{1}+M_{2}}^{*}(\hat{R}),
\end{aligned}
$$

where we have used the fact that

$$
\rho_{K_{1} 0}\left(\mathbf{r}_{1}\right)=\rho_{K_{1} 0}\left(r_{1}\right) Y_{L_{1} M_{1}}\left(\mathbf{r}_{1}\right)
$$

and the definition of the Bessel-Fourier transform

$$
\bar{\rho}_{K_{1} 0}(p)=4 \pi \int_{0}^{\infty} r_{1}^{2} d r_{1} j_{L_{1}}\left(p r_{1}\right) \rho_{K_{1} 0}\left(r_{1}\right) \text {. }
$$

We have denoted with $j_{l}$ and $Y_{l m}$ the Bessel and spherical harmonic functions, respectively. The caret over the spin variables denotes $\widehat{J}=\sqrt{2 J+1}$, and $\widetilde{v}(p)$ is the Fourier transform of $v$. According to Eq. (4) we can write $\mathbf{R}=\boldsymbol{\rho}+\frac{1}{2} \mathbf{s}$; then in this case we have [Eq. (6.50)] of Ref. [23]:

$$
R^{J} Y_{J M}^{*}(\hat{R})=\sqrt{4 \pi} \sum_{\lambda=0}^{J} \frac{1}{\hat{\lambda}}\left[\begin{array}{c}
2 L+1 \\
2 \lambda
\end{array}\right]^{1 / 2} \rho^{L-\lambda}\left(\frac{1}{2} s\right)^{\lambda} \sum_{\mu}\langle J-\lambda M-\mu \lambda \mu \mid J M\rangle Y_{J-\lambda M-\mu}^{*}(\hat{\rho}) Y_{\lambda \mu}^{*}(\hat{s})
$$


With the help of this equation we can write Eq. (A1) in the form

$$
\begin{aligned}
\Delta \mathcal{V}_{12}(\rho, \mathbf{s})= & \frac{1}{4 \pi^{4}} \sum_{K_{1} K_{2}} G_{K_{1} K_{2}}(\rho, s)(-)^{L_{1}-L_{2}} \hat{L}_{1}^{2} \hat{L}_{2}^{2} \\
& \times \sum_{J J^{\prime}} \frac{i^{-J+J^{\prime}}}{\hat{J} \hat{J}^{\prime}} \mathcal{R}_{K_{1} K_{2}}^{J}(\rho, s) \mathcal{R}_{K_{1} K_{2}}^{J^{\prime}}(\rho,-s)\left\langle L_{1} 0 L_{2} 0 \mid J 0\right\rangle\left\langle L_{1} 0 L_{2} 0 \mid J^{\prime} 0\right\rangle \\
& \times \sum_{\lambda=0}^{J}\left|\rho+\frac{1}{2} s\right|^{-J} \rho^{J-\lambda}\left(\frac{1}{2} s\right)^{\lambda}\left(\begin{array}{c}
2 J+1 \\
2 \lambda
\end{array} \sum_{\lambda^{\prime}=0}^{1 / 2}(-)^{\lambda^{\prime}}\left|\rho-\frac{1}{2} s\right|^{-J^{\prime}} \rho^{J^{\prime}-\lambda^{\prime}}\left(\frac{1}{2} s\right)^{\lambda^{\prime}}\left[\begin{array}{c}
2 J^{\prime}+1 \\
2 \lambda^{\prime}
\end{array}\right]^{1 / 2}\right. \\
& \times \sum_{\mu \mu^{\prime} M_{1} M_{2}} Y_{J-\lambda, M_{1}+M_{2}+\mu}(\hat{\rho}) Y_{\lambda-\mu}(\hat{s}) Y_{J^{\prime}-\lambda^{\prime}, M_{1}+M_{2}-\mu^{\prime}(\hat{\rho}) Y_{\lambda^{\prime} \mu^{\prime}}^{*}(\hat{s})} \\
& \quad \times\left\langle J-\lambda-\left(M_{1}+M_{2}+\mu\right) \lambda \mu \mid J-\left(M_{1}+M_{2}\right)\right\rangle\left\langle L_{1} M_{1} L_{2} M_{2} \mid J\left(M_{1}+M_{2}\right)\right\rangle \\
& \quad \times\left\langle J^{\prime}-\lambda^{\prime}-\left(M_{1}+M_{2}-\mu\right) \lambda^{\prime} \mu^{\prime} \mid J^{\prime}\left(M_{1}+M_{2}\right)\right\rangle\left\langle L_{1} M_{1} L_{2} M_{2} \mid J^{\prime}\left(M_{1}+M_{2}\right)\right\rangle,
\end{aligned}
$$

where we have defined

$$
\mathcal{R}_{K_{1} K_{2}}^{J}(\rho, s)=\int d p p^{2} \widetilde{v}(p) \bar{\rho}_{K_{1} 0}(p) \bar{\rho}_{0 K_{2}}(p) j_{J}\left(p\left|\rho+\frac{1}{2} s\right|\right) .
$$

The angular part can be easily worked out, and one finally gets

$$
\begin{aligned}
& \Delta \mathcal{V}_{12}(\rho, \mathbf{s})=\frac{1}{(2 \pi)^{6}} \sum_{K_{1} K_{2}} G_{K_{1} K_{2}}(\rho, s) \hat{L}_{1}^{2} \hat{L}_{2}^{2} \\
& \times \sum_{J}(-)^{J} \mathscr{R}_{K_{1} K_{2}}^{J}(\rho, s) R_{K_{1} K_{2}}^{J}(\rho,-s)\left\langle L_{1} \quad 0 L_{2} \quad 0 \mid J \quad 0\right\rangle 2\left(\left|\rho+\frac{1}{2} s\right|\left|\rho-\frac{1}{2} s\right|\right)^{-J} \rho^{2 J} \\
& \times \sum_{\lambda=0}^{J}\left(\frac{s}{2 \rho}\right)^{\lambda}\left[\begin{array}{c}
2 J+1 \\
2 \lambda
\end{array}\right)^{1 / 2} \widehat{(J-\lambda)} \sum_{\lambda^{\prime}=0}^{J^{\prime}}(-)^{\lambda^{\prime}}\left(\frac{s}{2 \rho}\right)^{\lambda^{\prime}}\left[\begin{array}{c}
2 J+1 \\
2 \lambda^{\prime}
\end{array}\right)^{1 / 2} \widehat{\left(J-\lambda^{\prime}\right)} \\
& \times \sum_{f}(-)^{f} W\left(\lambda, J-\lambda, \lambda^{\prime}, J-\lambda^{\prime} ; J f\right)\left\langle J-\lambda 0 J-\lambda^{\prime} 0 \mid f 0\right\rangle\left\langle\lambda 0 \lambda^{\prime} 0 \mid f 0\right\rangle P_{f}(\cos \theta) .
\end{aligned}
$$

where $W$ is the Racah coefficient and the $P_{f}(\cos \theta)$ are the Legendre polynomials; $\theta$ is the angle between $\rho$ and s.

An analogous expression is valid for $\Delta \mathcal{V}_{1}(\rho, \mathbf{s})$ and for $\Delta \mathcal{V}_{2}(\rho, \mathbf{s})$ :

$$
\begin{aligned}
\Delta \mathcal{V}_{1}(\rho, \mathbf{s})= & \frac{1}{16 \pi^{5}} \sum_{K_{1}} G_{K_{1} 0}(\rho, s) \mathcal{R}_{K_{1} 0}^{L_{1}}(\rho, s) \mathcal{R}_{K_{1} 0}^{L_{1}}(\rho,-s) \hat{L}_{1}^{2}(-)^{L_{1}} \\
& \times\left(\left|\rho+\frac{1}{2} s\right|\left|\rho-\frac{1}{2} s\right|\right)^{-L_{1}} \rho^{2 L_{1}} \sum_{\lambda=0}^{L_{1}}\left[\frac{s}{2 \rho}\right)^{\lambda}\left(\begin{array}{c}
2 L_{1}+1 \\
2 \lambda
\end{array}\right]^{1 / 2} \underbrace{L_{1}}_{\left.L_{1}-\lambda\right)}\left(-\sum_{\lambda^{\prime}=0}^{\lambda^{\prime}}\left[\frac{s}{2 \rho}\right]^{\lambda^{\prime}}\left(\begin{array}{c}
2 L_{1}+1 \\
2 \lambda^{\prime}
\end{array}\right]_{\left(L_{1}-\lambda^{\prime}\right)}^{1 / 2}\right. \\
& \times \sum_{f}(-)^{f} W\left(\lambda, L_{1}-\lambda, \lambda^{\prime}, L_{1}-\lambda^{\prime} ; L_{1} f\right)\left\langle L_{1}-\lambda 0 L_{1}-\lambda^{\prime} 0 \mid F 0\right\rangle\left\langle\lambda 0 \lambda^{\prime} 0 \mid f 0\right\rangle P_{f}(\cos \theta)
\end{aligned}
$$

[1] G. R. Satchler and W. G. Love, Phys. Rep. 55, 183 (1979).

[2] H. Baeza, B. Bilwes, R. Bilwes, J. Diaz, and J. L. Ferrero, Nucl. Phys. A419, 412 (1984); J. S. Lilley, B. R. Fulton, M. A. Nagarajan, I. J. Thompson, and D. W. Banes, Phys. Lett. B 151, 181 (1985); B. R. Fulton, D. W. Banes, J. S. Lilley, M. A. Nagarajan, and I. J. Thompson, ibid. 162, 55 (1985).

[3] M. A. Nagarajan, C. Mahaux, and G. R. Satchler, Phys. Rev. Lett. 54, 1136 (1985).

[4] C. Mahaux, G. R. Satchler, and H. Ngô, Nucl. Phys. A449, 354 (1986); ibid. A456, 134 (1986).

[5] R. Broglia, G. Pollarolo, and A. Winther, Nucl. Phys. A361, 307 (1981); G. Pollarolo, R. Broglia, and A. Winther, ibid. A406, 369 (1983); C. H. Dasso, S. Landowne, G. Pollarolo, and Aa. Winther, ibid. A459, 134 (1986).
[6] D. M. Brink and Fl. Stancu, Phys. Rev. C 30, 1904 (1984).

[7] M. V. Andrés, F. Catara, Ph. Chomaz, and E. G. Lanza, Phys. Rev. C 39, 99 (1989); Ph. Chomaz, Y. Blumenfeld, M. V. Andrés, F. Catara, and E. G. Lanza, Europhys. Lett. 8, 125 (1989).

[8] H. Feshbach, Ann. Phys. (N.Y.) 5, 357 (1958).

[9] N. Vinh Mau, Phys. Lett. B71, 5 (1977); Nucl. Phys. A457, 413 (1986); A470, 406 (1987); in Proceedings of Heavy Ion Collisions at Energies Near the Coulomb Barrier, Daresbury Laboratory, England, 1990, edited by M. A. Nagarajan (Institute of Physics and Physical Society, London, 1990), pp. 1-14.

[10] M. V. Andrés, F. Catara, and E. G. Lanza, Ref. [9], pp. 231-238.

[11] A. Bouyssy, N. Vinh Mau, and D. M. Brink, Phys. Lett. B 
102,109 (1981).

[12] A. Bouyssy, H. Ngô, and N. Vinh Mau, Nucl. Phys. A371, 173 (1981)

[13] F. G. Perey and B. Buck, Nucl. Phys. 32, 353 (1962); F. G. Perey and D. S. Saxon, Phys. Lett. 10, 107 (1964); R. Peierls and N. Vinh Mau, Nucl. Phys. A343, 1 (1980).

[14] Z. El-Itaoui, P. J. Ellis, and B. A. Mughrabi, Nucl. Phys. A441, 511 (1985); M. Lassaut, Phys. Lett. 105B, 252 (1981).

[15] N. V. Giai, Suppl. Prog. Theor. Phys. 74-75, 330 (1983); N. V. Giai, in Nuclear Collective Dynamcis, edited by D. Burucescu, V. Ceausescu, and N. V. Zamphir (World Scientific, Singapore, 1983), p. 356.

[16] J. P. Blaizot and D. Gogny, Nucl. Phys. A284, 429 (1977).

[17] R. Lipperheide and A. K. Schmidt, Nucl. Phys. A112, 65 (1968).

[18] I. J. Thompson, M. A. Nagarajan, J. S. Lilley, and M. J.
Smithson, Nucl. Phys. A505, 84 (1989).

[19] B. V. Carlson, T. Frederico, M. S. Hussein, H. Esbensen, and S. Landowne, Phys. Rev. C 41, 933 (1990).

[20] J. S. Eck, R. A. LaSalle, and D. Robinson, Phys. Rev. 186, 1132 (1969); K. O. Groeneveld, L. Meyer-Schutzmeister, A. Richter, and U. Strohbusch, Phys. Rev. C 6, 805 (1972); K. E. Rehm, W. Henning, J. R. Erskine, and D. G. Kovar, Phys. Rev. Lett. 40, 1479 (1978); S. Kubono, P. D. Bond, and C. E. Thorn, Phys. Lett. 81B, 140 (1979).

[21] S. E. Vigdor, D. G. Kovar, P. Sperr, J. Mahoney, A. Mechaca-Rocha, C. Olmer, and M. S. Zisman, Phys. Rev. C 20, 2147 (1979).

[22] H. Doubre, J. C. Jacmart, E. Plagnol, N. Poffé, M. Riou, and J. C. Roynette, Phys. Rev. C 15, 693 (1977).

[24] G. R. Satchler, Direct Nuclear Reactions (Oxford University Press, Oxford, 1983). 